\title{
Senescence-associated secretory phenotype favors the emergence of cancer stem-like cells
}

\author{
J Cahu* ${ }^{* 1}$, S Bustany ${ }^{1}$ and B Sola ${ }^{1}$
}

The molecular mechanisms underlying cancer resistance remain elusive. One possible explanation is that cancer stem cells (CSCs) elude drug treatment, emerge and reproduce a tumor. Using multiple myeloma as a paradigm, we showed that cancer stem-like cells (CSLCs) appear after genotoxic stress because of their intrinsic properties. However, these properties do not drive the emergence of the CSLCs. Following genotoxic stress, remaining DNA damages lead to a senescence-associated secretory phenotype (SASP). Senescent cells, which are the non-CSLCs, secrete chemokines contributing to the emergence, maintenance and migration of CSLCs. Downregulation of checkpoint protein 2, a key player of SASP, significantly reduced the emergence of CSLCs. Our results unravel a novel molecular mechanism by which SASP might promote malignancy, underlining the dual role of senescence in tumorigenesis. This mechanism, based on mutual cooperation among tumor cells, illustrates how cancer may relapse; its targeting could represent new therapeutic opportunities.

Cell Death and Disease (2012) 3, e446; doi:10.1038/cddis.2012.183; published online 20 December 2012

Subject Category: Cancer

Despite the development of new therapeutic strategies, cancer remains difficult to cure. Resistance of cancer cells may account for this difficulty. This resistance has been shown to rely on cancer stem cells (CSCs). CSCs are thought to represent a minority of cells within a tumor. In contrast to most of the tumor cells, they are able to self-renew, to differentiate and thus to reproduce a tumor. ${ }^{1}$ In addition, they are particularly resistant to anticancer therapies. While the chemoresistance of CSCs relies on efficient drug efflux, radioresistance is due to intrinsic properties enabling them to counteract the formation of DNA double-strand breaks (DSBs) ${ }^{2-5}$ Resistant CSCs remaining after anticancer therapies could then emerge and repopulate the tumor bulk leading to cancer relapse.

Multiple myeloma (MM) is a peculiar blood cancer as most anticancer therapies lead to nearly complete disappearance of the tumor bulk but not to long-term survival. ${ }^{6,7} \mathrm{MM}$ is therefore characterized by a high percentage of relapse, suggesting a role for CSCs. We have studied the response of MM cells towards genotoxic stress-inducing DSB (X-irradiation or doxorubicin) and uncovered a new mechanism responsible for the emergence of cancer stem-like cells (CSLCs).

\section{Results}

Genotoxic stress induces the emergence of a cell population that resembles CSCs. MM is characterized by the accumulation of malignant plasma cells in the bone marrow. Plasma cells are terminally differentiated cells of the $B$ lymphoid lineage. They express the cell surface marker CD138 (cluster of differentiation 138), whereas less differentiated B cells do not. The CSC of MM has been described as being within the $\mathrm{CD} 138^{\text {low }}$ population of cells and further characterized as CD45 $5^{\text {high }} / \mathrm{CD} 20^{\text {high }}$ mature B cells. ${ }^{8-13}$

We exposed MM cells (RPMI 8226, the reference MM cell line) to X-irradiation and followed the CD138 ${ }^{\text {low }} / C D 45^{\text {high }} /$ CD20high cell population by flow cytometry (Supplementary Figure S1a). Importantly, special care was taken to remove apoptotic MM cells before cytometry analysis because such cells loose the CD138 membrane marker and could therefore bias the analysis. We observed a significant increase in the CD138 ${ }^{\text {low}} / C D 45^{\text {high }} / C D 20^{\text {high }}$ cell population 7 days after a dose of 6 Gy (Figure 1a, left panel). To insure that this increase was not treatment-specific, we exposed MM cells to doxorubicin, an intercalating agent. The percentage of CD138 ${ }^{\text {low }} / C D 45^{\text {high }} / C D 20^{\text {high }}$ cells also increased in a dose-dependent manner 2 days after doxorubicin treatment (Figure 1a, right panel). These results show that both $X$-irradiation and doxorubicin treatment select for a population of $\mathrm{CD} 138^{\text {low }} / \mathrm{CD} 45^{\text {high }} / \mathrm{CD} 20^{\text {high }}$ cells, which phenotypically resemble MM CSCs. ${ }^{8,13}$

To ensure for the enrichment of CD138 ${ }^{\text {low }} / C D 45^{\text {high }} / C D 20^{\text {high }}$ cells, we used quantitative reverse transcription-polymerase

\footnotetext{
${ }^{1}$ MILPAT (EA 4652), Faculté de Médecine, Université de Caen Basse-Normandie, CHU Côte de Nacre, Caen, France

*Corresponding author: J Cahu, MILPAT (EA 4652), Faculté de médecine, Université de Caen Basse-Normandie, CHU Côte de Nacre, Caen 14032, France. Tel: + 332310682 25; Fax: + 332310682 24; E-mail: julie.cahu@ gmail.com

Keywords: cancer stem cell; senescence; chemokines; cell cooperation

Abbreviations: CSCs, cancer stem cells; MM, multiple myeloma; CSLCs, cancer stem-like cells; SASP, senescence-associated secretory phenotype; CHK2, checkpoint protein 2; DSBs, double-strand breaks; CD, cluster of differentiation; qRT-PCR, quantitative reverse transcription-polymerase chain reaction; G2, gap 2; M, mitosis; G0, gap 0; G1, gap 1; ROS, reactive oxygen species; DCFDA, dichlorodihydrofluorescein diacetate; ATM, ataxia telangiectasia mutated; ATR, ataxia telangiectasia mutated and Rad3 related; $\mathrm{C}_{12} \mathrm{FDG}$, 5-dodecanoylaminofluorescein di- $\beta$-D-galactopyranoside; IL-6, interleukin-6; IL-8, interleukin-8; IP-10, interferon $\gamma$ induced protein 10; CXCL10, chemokine (C-X-C motif) ligand 10; RANTES, regulated and normal T-cell expressed and secreted; CCL5, chemokine (C-C motif) ligand 5; FCS, fetal calf serum; PBS, phosphate-buffered saline; FITC, fluorescein isothiocyanate; IgG, immunoglobulin G; PE, phycoerythrin; PC5, phycoerythrin cyanin 5; $\mathrm{EtOH}$, ethanol; BSA, bovine serum albumin; GAPDH, glyceraldehyde 3-phosphate dehydrogenase; shRNA, short hairpin RNA

Received 05.7.12; revised 31.10.12; accepted 05.11.12; Edited by Y Shi
} 
a
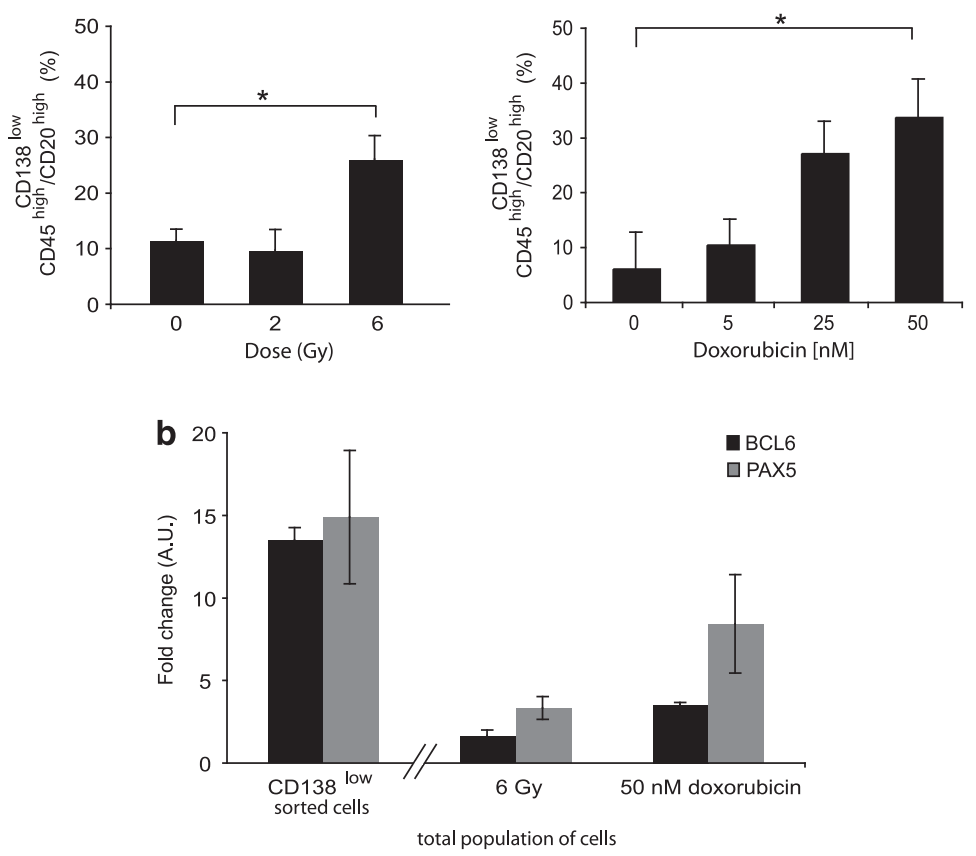

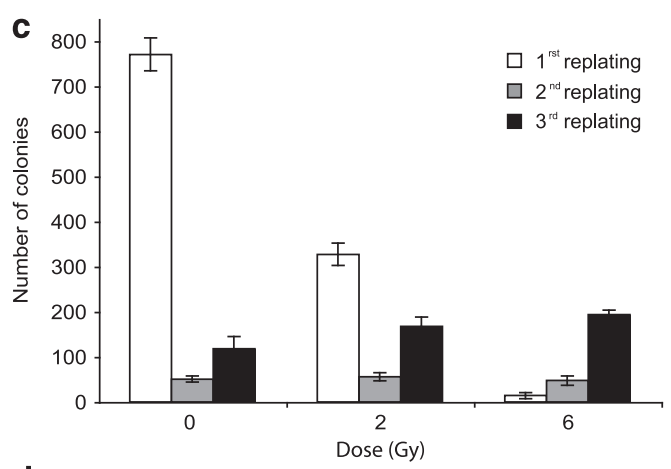

d
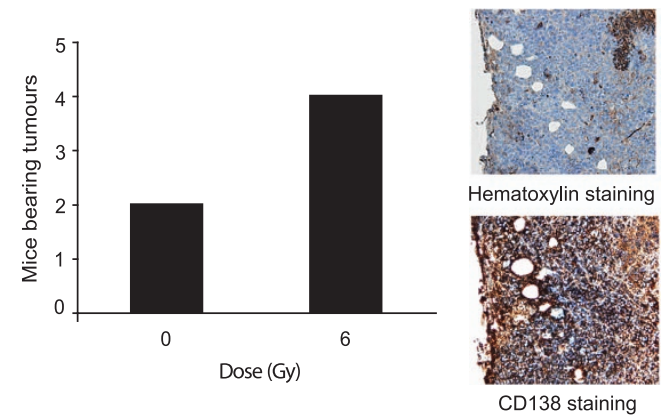

Figure 1 Genotoxic stress induces the emergence of a cell population that resembles CSCs. (a) Flow cytometry analysis of RPMI 8226 cells 7 days after X-irradiation (0-6 Gy) and 2 days after doxorubicin treatment ( $0-50 \mathrm{nM})$ are presented on the left $(P=0.014)$ and on the right $(P=0.0011)$, respectively. (b) QRT-PCR analysis of $B C L 6$ and PAX5 gene expressions of CD138-sorted RPMI 8226 cells and RPMI 8226 cells 7 days after X-irradiation (6 Gy) and 2 days after doxorubicin treatment (50 nM); the untreated main population served as reference. (c) RPMI 8226 cells were seeded in methylcellulose 7 days after X-ray treatment ( 6 Gy) or control ( 0 Gy) and the number of colonies was quantified after the first, second and third replating. (d) At 7 days after X-ray treatment, $10^{4}$ irradiated (6 Gy) or control (0 Gy) RPMl 8226 cells were injected subcutaneously into five nude mice per series. The graphic illustrates the number of mice bearing tumors 12 weeks post-injection. Tumors were stained with hematoxylin or labeled with anti-CD138 antibody. Images were taken at $\times 40$ magnification. Data are means \pm S.D. from at least three independent experiments; ${ }^{*} P<0.05$; a.u., arbitrary unit

chain reaction (qRT-PCR) to quantify the expression of the B-cell genes BCL6 and of PAX5. The expression of both genes was higher in sorted CD138 ${ }^{\text {low }}$ cells and in cells after genotoxic stress than in the main population (Figure 1b). This result indicates that cells emerging after genotoxic stress genetically resemble mature B cells. A clonogenic test was used to determine whether cells emerging after genotoxic stress could self-renew. While the clonogenic capacity of control non-irradiated cells decreased with the number of replating, the clonogenic capacity of cells 7 days after $6 \mathrm{~Gy}$ irradiation increased, suggesting that irradiated cells have a higher clonogenic capacity than non-irradiated cells (Figure 1c). Similar results were obtained after doxorubicin treatment (Supplementary Figure S1b). Moreover, 7 days after $\mathrm{X}$-irradiation with $6 \mathrm{~Gy}, 10^{4}$ cells were injected subcutaneously into nude mice and the presence of tumor was monitored. More mice developed tumors when injected with irradiated cells than control non-irradiated cells (Figure 1d). Immunohistological staining revealed that tumors originating from irradiated cells were constituted by CD138 ${ }^{\text {high }}$ plasma cells, showing that cells resisting irradiation could reproduce the original tumor. Thus, cells emerging after genotoxic stress have self-renewal and differentiation capacities. These findings are consistent with CD138 low/ $\mathrm{CD} 45^{\text {high }} / \mathrm{CD} 20^{\text {high }}$ emerging cells having characteristics and properties of CSLCs.
Emergence of CSLCs is, in part, due to their intrinsic properties of resistance. We then seek to understand how CSLCs emerge. We tested whether the CD138 ${ }^{\text {low }}$ population has intrinsic resistance to genotoxic stress, thereby favoring its subsequent emergence from the main population. We used flow cytometry to analyze the cell cycle distribution of CD138 high and CD138 ${ }^{\text {low }}$ cells subjected to $6 \mathrm{~Gy} \mathrm{X}$-irradiation. CD138 $8^{\text {high }}$ cells were arrested at the G2-M phase 2 days after treatment, whereas CD138 ${ }^{\text {low }}$ cells did not show any cell cycle arrest (Figure 2a, left panel). Furthermore, and in contrast to CD138 high cells, the percentage of CD138 $8^{\text {low }}$ cells in the G0-G1 phase remained stable after X-rays over the course of the experiment (Figure 2a, right panel). Thus, $\mathrm{CD} 138^{\text {low }}$ cells escape cell cycle arrest induced by X-irradiation, suggesting intrinsic resistance of these cells.

Low intracellular concentration of reactive oxygen species (ROS) has been shown to contribute to radioresistance. ${ }^{5}$ To measure the intracellular production of ROS, CD138-sorted cells were labeled with dichlorodihydrofluorescein diacetate (DCFDA) and analyzed by flow cytometry (Figure 2b, left panel). CD138 ${ }^{\text {low }}$ cells were significantly less stained than $\mathrm{CD} 138^{\text {high }}$ cells (Figure $2 \mathrm{~b}$, right panel), which was an evidence that CD138 ${ }^{\text {low }}$ cells produce intrinsically less ROS than $\mathrm{CD} 138^{\text {high }}$ cells.

Lower ROS availability is expected to be associated with less DNA damage. DNA damages, triggered by genotoxic 
a

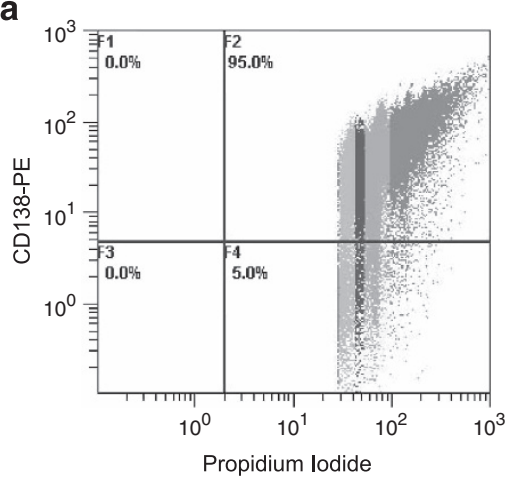

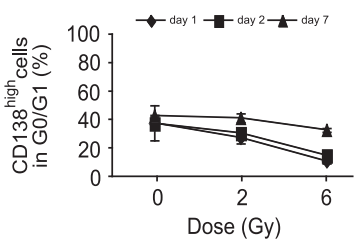

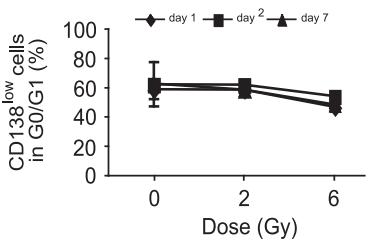

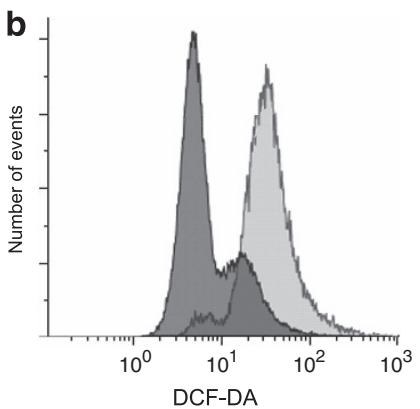

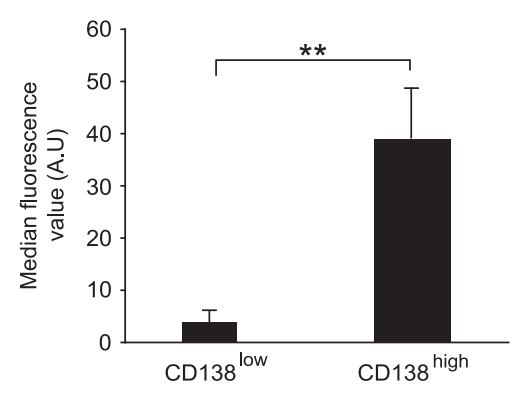

C

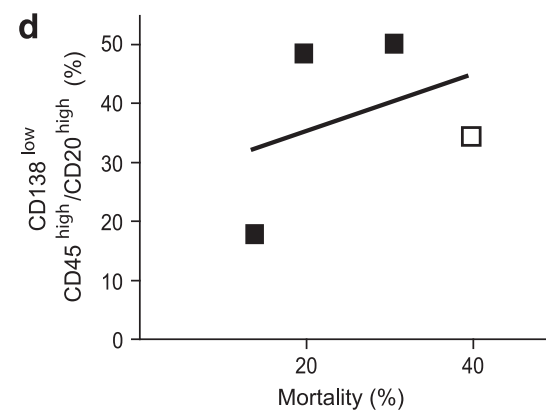

Figure 2 Emergence of CSLCs is, in part, due to their intrinsic properties. (a) Cell cycle distribution of CD138 $8^{\text {low }}$ and CD $138^{\text {high }}$ RPMl 8226 cells 2 days after a 6 Gy dose of X-irradiation is illustrated on the left and the percentage of cells in G0/G1 after flow cytometry analysis on the right. (b) Intracellular ROS were detected by DCFDA staining in $\mathrm{CD} 138^{\text {low }}$ (dark gray) and CD138 ${ }^{\text {high }}$ (light gray) cells. Percentage of DCFDA-positive cells is represented on the graph $(P=0.00088)$. (c) Irradiated (6 Gy) and control cells (0 Gy) were double-stained for CD138 and $\gamma \mathrm{H} 2 \mathrm{AX} 30 \mathrm{~min}$ after X-irradiation, and quantitatively analyzed by flow cytometry. (d) Percentage of mortality is plotted against the percentage of $\mathrm{CD} 138^{\text {low }} / \mathrm{CD} 45^{\text {high }} / \mathrm{CD} 20^{\text {high }}$ cells $\left(R^{2}=0.1358\right)$. White and black squares represent data from cells subjected to $\mathrm{X}$-irradiation $(6 \mathrm{~Gy}, 7$ days post $\mathrm{X}$-irradiation) and doxorubicin treatment (2 days post-treatment at 2.7, 13.5 and $50 \mathrm{nM}$ ), respectively. Data are means \pm S.D. from at least three independent experiments, except for (d) where squares represent the mean of two independent experiments; ${ }^{* *} P<0.001$

stress, activate the kinases, ataxia telangiectasia mutated/ ataxia telangiectasia mutated and Rad3 related (ATM/ATR), which phosphorylate histone $\mathrm{H} 2 \mathrm{AX}(\gamma \mathrm{H} 2 \mathrm{AX})$ at break sites facilitating their recognition by the DNA repair machinery. Complete repair allows cells to survive, whereas unrepaired breaks lead to apoptosis or cellular senescence. ${ }^{14}$ To test whether MM cells are less prone to DNA damage, cells were double-stained for CD138 and $\gamma \mathrm{H} 2 \mathrm{AX}$. Flow cytometry analysis revealed that $\gamma \mathrm{H} 2 \mathrm{AX}$ was phosphorylated in a dosedependent manner in CD138 ${ }^{\text {high }}$ cells, showing that X-irradiation induced stable DBSs. In contrast, the phosphorylation of $\gamma \mathrm{H} 2 \mathrm{AX}$ remained low in CD138 ${ }^{\text {low }}$ cells, irrespective of the dose of $X$-irradiation (Figure $2 \mathrm{c}$ ). This indicates that, unlike CD138 ${ }^{\text {high }}$ cells, CD138 ${ }^{\text {low }}$ cells are protected against DSB formation.
Although these results show that MM CSLCs have intrinsic properties allowing them to elude death-induced treatments, to our surprise, no direct correlation was found between cell mortality and the percentage of CD $138^{\text {low }} / C D 45^{\text {high }} / C D 20^{\text {high }}$ cells (Figure $2 \mathrm{~d}$ ). This result suggests that another mechanism contributes actively to the selection of CSCLs.

\section{SASP induced by genotoxic stress drives the emergence} of CSLCs. One consequence of genotoxic stress is the induction of cellular senescence. Indeed, $\beta$-galactosidase staining revealed the induction of senescence in MM cells upon genotoxic stress (Figure 3a). Similar results were obtained with the MM cell line $\mathrm{NCl}-\mathrm{H} 929$ harboring a wildtype TP53 gene, indicating that genotoxic stress-induced senescence is independent of TP53 status (data not shown). 
a
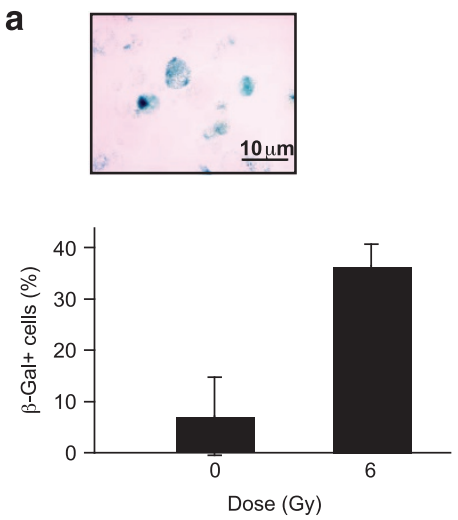

b

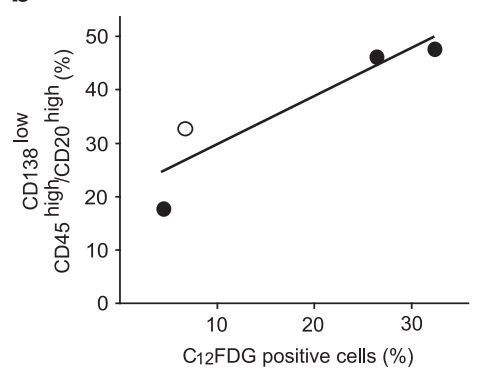

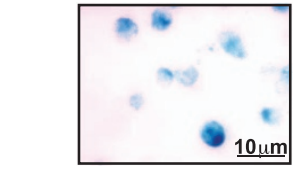

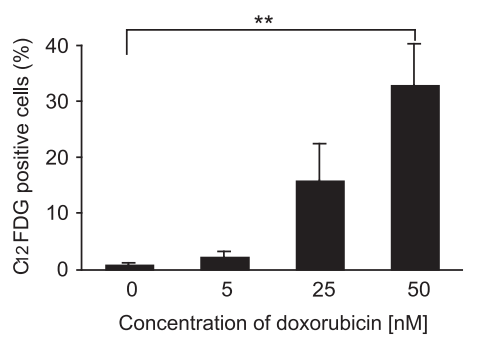

Concentration of doxorubicin [nM] c

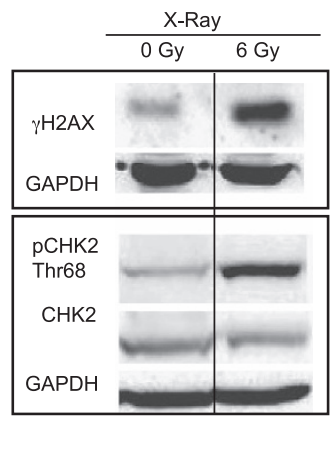

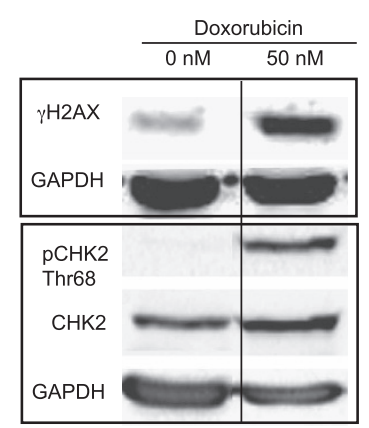
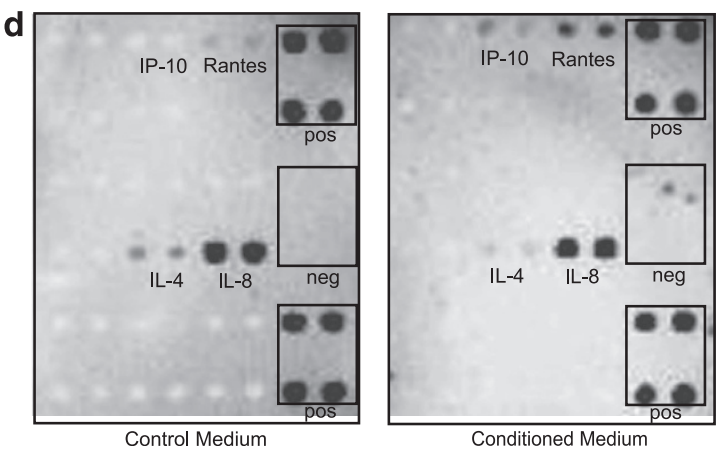

e

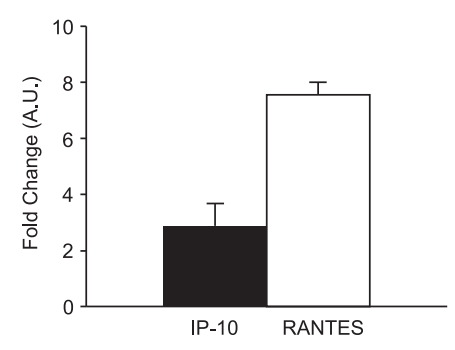

Figure 3 Emergence of CSLCs correlates with an SASP induced by genotoxic stress. (a) RPMI 8226 were X-irradiated with a dose of 6 Gy, stained by $\beta$-galactosidase 7 days post-treatment and quantified. For each experiment, several fields were counted. Data are means \pm S.D. for 50 cells counted over two independent experiments (left panel). RPMI 8226 cells were treated with doxorubicin $(50 \mathrm{nM})$ and stained with $\beta$-galactosidase 2 days post-treatment. Senescent cells were stained with $\mathrm{C}_{12} \mathrm{FDG}$ and quantified by flow cytometry 2 days post-treatment with doxorubicin $(2.7,13.5$ and $50 \mathrm{nM})(P=0.00062)$ (right panel). (b) Percentage of senescent cells is plotted against the percentage of $\mathrm{CD} 138^{\text {low }} / \mathrm{CD} 45^{\text {high }} / \mathrm{CD} 20^{\text {high }}$ cells $\left(R^{2}=0.8288\right)$. White and black circles represent data from cells subjected to $\mathrm{X}$-irradiation $(6 \mathrm{~Gy}, 7$ days post $\mathrm{X}$-irradiation) and doxorubicin treatment (2 days post-treatment at 5,25 and $50 \mathrm{nM}$ ), respectively. (c) Whole-cell lysates were prepared 7 days after X-irradiation with a dose of $6 \mathrm{~Gy}$ (left panel) or 2 days after a treatment with $50 \mathrm{nM}$ doxorubicin (right panel) and analyzed by western blot with anti- $\gamma \mathrm{H} 2 \mathrm{AX},-\mathrm{CHK} 2,-\mathrm{pCHK} 2$ (Thr68) and -GAPDH (as control) antibodies. Similar results were obtained in two independent experiments. (d) A cytokine array was used to identify the cytokines and/or chemokines released into the medium by cells treated with $50 \mathrm{nM}$ doxorubicin (conditioned medium). The medium of untreated cells was used as control (control medium). (e) QRT-PCR analysis of IP-10 and RANTES expressions in RPMI 82262 days after doxorubicin treatment $(50 \mathrm{nM})$; the untreated main population served as reference. Data are means \pm S.D. from at least three independent experiments, except for (b) where circles represent the mean of two independent experiments; ${ }^{* \star} P<0.001$

We used $\beta$-galactosidase staining or 5-dodecanoylaminofluorescein di- $\beta$-D-galactopyranoside $\left(\mathrm{C}_{12} \mathrm{FDG}\right)$, a membrane-permeable molecule that specifically stains senescent cells, to evaluate senescence. The number of senescent cells increased with $X$-irradiation dose and doxorubicin concentration (Figure 3a). Interestingly, X-irradiation was less effective than doxorubicin to induce senescence and the emergence of CSLCs, suggesting a link between these two processes. The percentage of $\mathrm{C}_{12}$ FDG-positive cells was therefore plotted against the percentage of CD138 ${ }^{\text {low }} / C D 45^{\text {high }} / \mathrm{CD} 20^{\text {high }}$ cells. In contrast to mortality (Figure 2d), the percentage of senescent cells correlated with the percentage of CSLCs (Figure 3b), suggesting that senescence actively selects for CSLCs.

Senescence occurs in various cellular contexts, including telomere shortening, oncogene activation and accumulation of DNA damage. ${ }^{15} \mathrm{X}$-irradiated and doxorubicin-treated cells were analyzed for the presence of remaining DNA breaks. Western blot analyses showed upregulation of $\gamma \mathrm{H} 2 \mathrm{AX}$ after genotoxic stress, which was an evidence that DNA breaks were still present (Figure 3c). It has shown that senescence due to remaining DNA DSBs is associated with unusual lasting phosphorylation of the protein checkpoint protein 2 (CHK2) by ATM/ATR and as well as the release of cytokines and/or chemokines. ${ }^{16,17}$ This senescence is referred as senescence-associated with secretory phenotype (SASP) ${ }^{18}$ Similarly, western blot analyses showed a lasting phosphorylation of CHK2 after $\mathrm{X}$-irradiation and doxorubicin treatment in our model (Figure 3c). We then performed a cytokine array to search for cytokines and/or chemokines released in the culture medium (hereafter referred as conditioned medium, i.e. medium of cells treated with $50 \mathrm{nM}$ doxorubicin for 2 days). None of the known SASP factors, ${ }^{17,18}$ such as interleukin-6 (IL-6) or interleukin-8 (IL-8), were particularly abundant; rather, chemokines, such as interferon $\gamma$-induced protein 10 (IP-10) (or chemokine (C-X-C motif) ligand 10 (CXCL10)) and regulated and normal $\mathrm{T}$-cell expressed and secreted (RANTES) (or chemokine (C-C motif) ligand 5 (CCL5)), were identified as being secreted by $\mathrm{MM}$ cells after doxorubicin treatment (Figure 3d). To confirm this result, the expression of IP-10 and RANTES was analyzed by qRT-PCR after doxorubicin treatment. Senescent MM cells express both chemokines (Figure 3e). Thus, after genotoxic stress, the 
emergence of CSLCs correlates with SASP, in which IP-10 and RANTES are released likely affecting the microenvironment of tumor cells.

Chemokines released by senescent non-CSLCs promote the emergence, maintenance and migration of CSLCs. RANTES has been shown to promote tumor growth in vivo and IP-10 to have an antiapoptotic effect and to stimulate the migration of MM cells. ${ }^{19,20}$ Therefore, chemokines released by senescent cells, such as RANTES or IP-10, might contribute to the selection of CSLCs after genotoxic stress. We used $\mathrm{C}_{12} \mathrm{FDG}$ and $\mathrm{CD} 138$ labeling to identify senescent cells. Flow cytometry analysis of double-stained cells indicated that senescent cells were CD138 ${ }^{\text {high }}$ cells, the non-CSLC population (Figure $4 a$ ). We then analyzed by qRTPCR the expression of both chemokines in doxorubicintreated and -sorted cells. Interestingly, we could show that IP-10 and RANTES are preferentially expressed in CD138 ${ }^{\text {high }}$ senescent cells (Figure 4b). To understand how chemokines released by non-CSLC population contribute to the emergence of CSLCs, we studied the effect of the conditioned medium on sorted CSLCs. The cytokine array we used tests 18 major cytokines and/or chemokines. Therefore, we cannot exclude that other secreted factors influence CSLCs. To circumvent this limitation, we exploited the differentiation properties of CSLCs. CD138-sorted cells were cultured either in the control medium (medium of untreated cells) or in the conditioned medium. CD138 ${ }^{\text {low }}$ cells cultured in the control medium differentiated very rapidly into CD138 ${ }^{\text {high }}$ cells. By contrast, when cultured in the conditioned medium, CD138 ${ }^{\text {low }}$ cells remained undifferentiated (Figure 4c). Findings with the $\mathrm{NCl}-\mathrm{H} 929$ cell line were entirely consistent, showing that the results were neither cell linespecific nor TP53-dependent (Supplementary Figure S2). These results show that chemokines released by senescent cells favor CSLCs' maintenance. They further reveal unexpected cooperation between CSLCs and non-CSLCs. Increased tumorigenesis might be associated with an increase in cancer cell migration. ${ }^{21}$ Having identified the chemokine RANTES as being secreted by non-CSLCs, we tested the migratory capacity of CSLC cells. MM cells were allowed to migrate for $24 \mathrm{~h}$; thereafter, migrating cells were collected and stained with CD138. Flow cytometry analysis reveals that, in contrast to CD138 ${ }^{\text {high }}$ cells, CD138 ${ }^{\text {low }}$ cells migrated significantly more towards the conditioned medium than towards the culture medium (Figure 4d). This suggests that CSLCs respond to chemokines released by senescent non-CSLCs and migrate. This result further reinforces the
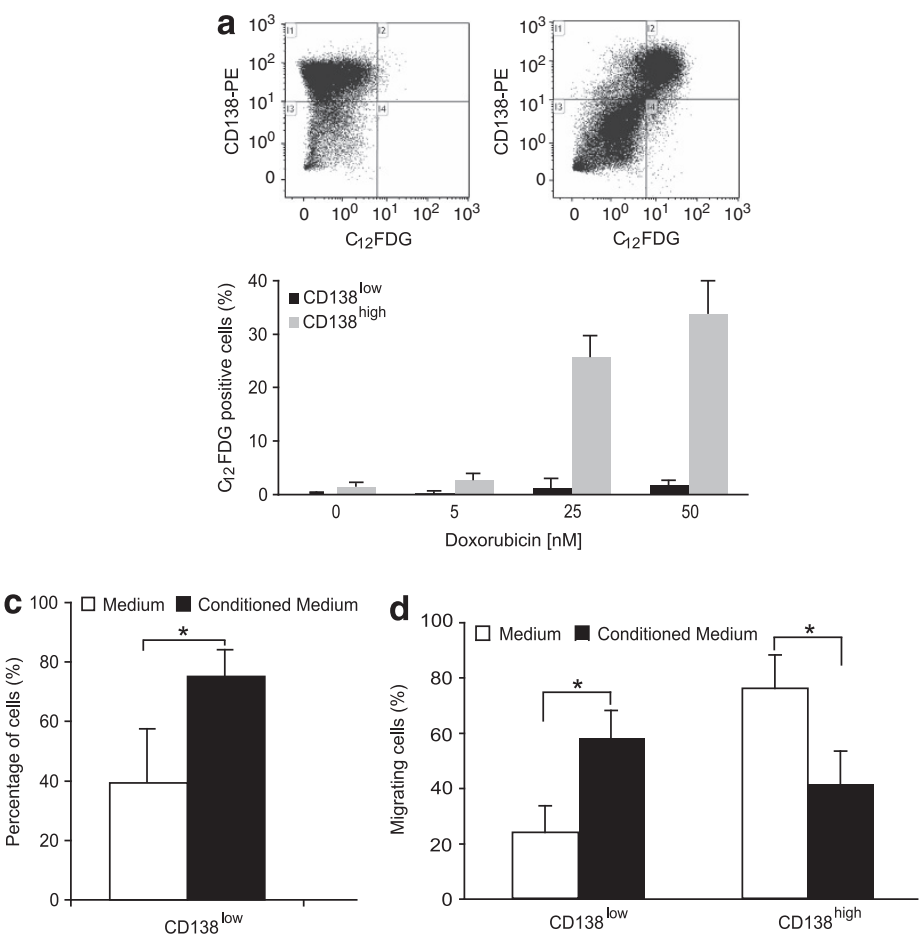
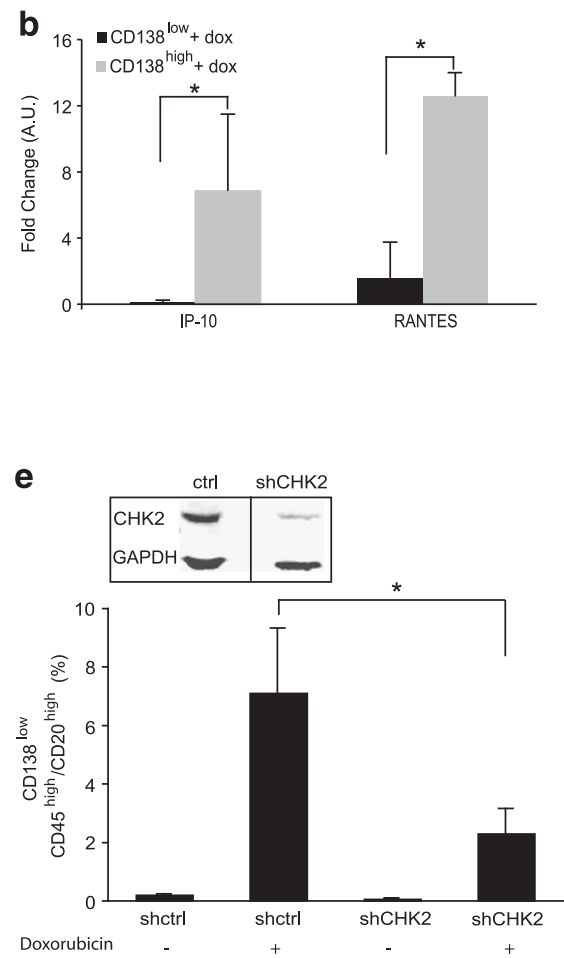

Figure 4 The SASP of non-CSLCs is essential for CSLCs. (a) RPMI 8226 cells treated with doxorubicin $(2.7,13.5$ and 50 nM) for 2 days were double-stained with CD138 and $\mathrm{C}_{12} \mathrm{FDG}$ as illustrated in the top panel for 0 and $50 \mathrm{nM}$ doxorubicin. CD138 ${ }^{\text {low }}$ and $\mathrm{CD} 138^{\text {high }}$ senescent cells were quantified by flow cytometry. (b) QRT-PCR analysis of IP-10 and RANTES expressions in CD138 ${ }^{\text {low }}$ - and CD $138^{\text {high }}$-sorted RPMI 8226 cells 2 days after doxorubicin treatment (50 nM); the untreated CD $138^{\text {low }}$ - and CD $138^{\text {high }}$ sorted RPMI 8226 cells served as reference ( $P=0.12$ and $P=0.0031$ for IP-10 and RANTES, respectively). (c) CD $138^{\text {low }}$-sorted RPMI 8226 cells were cultured either in the control medium (medium of untreated cells) or in the conditioned medium (medium of cells treated with $50 \mathrm{nM}$ doxorubicin for 2 days). Cells were stained with CD138 after 1 day of culture and analyzed by flow cytometry $(P=0.02)$. (d) RPMl 8226 cells having migrated overnight towards the control medium or the conditioned medium were analyzed by flow cytometry after CD138 labeling $\left(P=0.0047\right.$ and $P=0.0045$ for CD138 $8^{\text {low }}$ and CD138 high, respectively). (e) RPMI 8226 cells were infected with lentiviral particles containing shRNA-targeting CHK2 or control shRNA. Whole-cell lysates were prepared and analyzed by western blot with anti-CHK2 antibody to confirm the downregulation of CHK2. Cells were treated (or not for controls) with doxorubicin $(50 \mathrm{nM})$ for 2 days and $\mathrm{CD} 138^{\mathrm{low}} / \mathrm{CD} 45^{\text {high }} / \mathrm{CD} 20^{\text {high }}$ cells were tracked and quantified as described previously $(P=0.049)$. Data are means \pm S.D. from at least three independent experiments; ${ }^{*} P<0.5$ 
existence of cooperation between CSLC and non-CSLC populations.

Rodier et al. ${ }^{17}$ have shown that CHK2 is necessary for the establishment of SASP. To investigate if the senescence associated with persistent DNA damages and the secretion of cytokines have a role in the emergence of CSLCs, the expression of $\mathrm{CHK} 2$ was downregulated using lentivirus delivering short hairpin RNA (shRNA). The downregulation of $\mathrm{CHK} 2$ significantly decreased the percentage of CD138 ${ }^{\mathrm{low}} /$ CD45 ${ }^{\text {high }} / \mathrm{CD} 20^{\text {high }}$ cells emerging after doxorubicin treatment (Figure 4e). CHK2 was downregulated in the whole population. We cannot exclude that loss of $\mathrm{CHK} 2$ by itself might affect the emergence of the CD138 ${ }^{\text {low }}$ population. Nevertheless, altogether these results suggest that chemokines released by senescent non-CSLCs are essential for the emergence of CSLCs after genotoxic stress and further identifies CHK2 as an essential player.

\section{Discussion}

We described here the emergence of a population of cancer cells from the tumor bulk after genotoxic stress. These cells resemble MM CSCs. They display the cell surface proteins CD $138^{\text {low }} / C D 45^{\text {high }} / C D 20^{\text {high }}$ and express B-cell marker genes, including BCL6 and PAX5. Using an in vitro clonogenic assay and an in vivo xenograft model, we showed that emerging cells possess self-renewal and differentiation properties characteristic of CSCs. The precise identity of MM CSC remains controversial, probably due to the diversity of models and techniques used. ${ }^{8-13} \mathrm{Kim}$ et al. ${ }^{22}$ have recently identified the CD138 $8^{\text {high }} / \mathrm{CD} 38^{\text {high }} / \mathrm{CD} 45^{\text {low }} / \mathrm{CD} 19^{\text {low }}$ population as being enriched for tumorigenic myeloma cells. ${ }^{22}$ We cannot exclude that this population also contains 'stem cell activity'. However, independently of the model used, the population of $\mathrm{CD}_{138^{\text {low }}}$ cells, initially described as MM CSCs, ${ }^{8-10}$ appears unanimously more resistant to drugs than the main population of CD138 ${ }^{\text {high }}$ cells. ${ }^{11,13}$ Our results are consistent with the previous results showing that MM cells resistant to anticancer therapies are within the CD138 low population $^{10,13}$ and further extend this resistance to drugs inducing senescence.

We further showed that resistance of MM CSLCs relies on intrinsic properties shared with breast and glioma CSCs, such as low intracellular ROS level and low generation of DSBs, respectively. ${ }^{3-5}$ However, these intrinsic properties initiate but do not drive the emergence of CSLCs after genotoxic stress. In contrast to Bao et al., ${ }^{4}$ we did not observe a significant increase in the percentage of CSLCs 2 days after X-irradiation, suggesting that the mechanisms promoting the emergence of CSLCs might differ between cancers. ${ }^{4}$ Rather, senescence and a significant increase in the percentage of CSLCs appeared concomitantly 7 days after a dose of 6 Gy of $X$-rays. Similarly, doxorubicin-induced senescence paralleled the emergence of CSLCs in a dose-dependent manner, suggesting that senescence actively selects for CSLCs. In both cases, senescence was triggered by persistent DNA damages, accompanied by a sustained activation of $\mathrm{CHK} 2$ and release of chemokines, all characteristic of SASP. ${ }^{16,17}$

Known chemokines associated with SASP, such as IL-6, were not released in substantial amount by senescent cells.
However, we identified the chemokines IP-10 and RANTES. RANTES has already been reported to be secreted by senescent fibroblasts. ${ }^{23}$ Moreover, Karnoub et al. ${ }^{21}$ have shown that RANTES enhances motility, invasion and metastasis of breast cancer cells. Interestingly, it has been proposed that CSCs could have a role in metastasis, ${ }^{24}$ and we could show that MM CSLCs migrate preferentially towards medium containing chemokines released by senescent cells, likely attracted by RANTES. The downregulation of CHK2, a key player for SASP, prevents the emergence of CSLCs, suggesting that SASP has a role in this process.

Senescence has been reported to either limit or promote tumorigenesis. ${ }^{19,23,25}$ Coppé et al. ${ }^{26}$ have shown that SASP can promote malignancy by inducing an epithelial-tomesenchymal transition, a process that has been linked to the acquisition of stemness properties by cancer cells. We showed that mice injected with irradiated cells have a higher chance to develop tumors. This supports the idea that SASP promotes tumorigenesis albeit by an original mechanism involving the emergence of CSLCs. We envisage the emergence of CSLCs as a very dynamic process related to microenvironmental cues. It will be interesting to know if CSLCs arise from the outgrowth of a reservoir of CSLCs and/ or from the dedifferentiation of non-CSLCs.

The release of chemokines by the tumor microenvironment has been described to contribute to cancer development. ${ }^{27,28}$ Here, we identified senescent cells as the CSLC counterpart and showed that within the same tumor, chemokines released by non-CSLC senescent cells not only favor the emergence of CSLCs but also their maintenance and migration. These three steps, emergence, maintenance and migration, illustrate how SASP shapes the behavior of CSLCs to promote malignancy. Interestingly, they also shed light on an unexpected cooperation between non-CSLCs and CSLCs. This cooperation can be refined as mutualism. ${ }^{29}$ Senescent non-CSLCs support CSLCs, which will eventually differentiate into non-CSLCs, thereby reconstructing the tumor bulk. Thus, both cell populations, non-CSLCs and CSLCs, benefit from this cooperation.

Anticancer therapies based on genotoxic stress may induce senescence of cancer cells. We unravel here a mechanism based on cell cooperation by which SASP promotes tumorigenesis, underlining the dual role of senescence in tumorigenesis. We further identified $\mathrm{CHK} 2$ as a major actor for the emergence of CSLCs, opening new therapeutic opportunities.

\section{Materials and Methods}

Cell culture. RPMI 8226 and $\mathrm{NCl} H 929 \mathrm{MM}$ cells were obtained from R Bataille (Center de Recherche en Cancérologie, Nantes-Angers, France). Cells were cultured at $37^{\circ} \mathrm{C}$ under $5 \% \mathrm{CO}_{2}$ in RPMl 1640 medium (Lonza SPRL, Verviers, Belgium) supplemented with $10 \%$ fetal calf serum (FCS; PAA Laboratories $\mathrm{GmbH}$, Pashing, Austria), $2 \mathrm{mM}$ L-glutamine and antibiotics (Lonza).

Cell viability. Cell viability was followed using Trypan blue exclusion tests. Briefly, cells were mixed 1:1 with Trypan blue (vol/vol) and filled into a Malassez chamber. Blue cells were counted as dead cells, whereas white cells were counted as alive.

Flow cytometry analyses. For membrane staining, cells were labeled following the kit as per the manufacturer's instructions. Briefly, cells were suspended in complete RPMI 1640 medium and incubated with the fluorescent 
antibody for $15 \mathrm{~min}$ at $4^{\circ} \mathrm{C}$, washed and resuspended in phosphate-buffered saline (PBS). The following antibodies and isotypic controls were used: CD45-fluorescein isothiocyanate (FITC) and immunoglobulin G (IgG)1a-FITC; CD138-phycoerythrin (PE) and IgG1a-PE; CD20-phycoerythrin cyanin 5 (PC5) and IgG2a-PC5 (Beckman Coulter, Villepinte, France); and CD45-Vybrant blue and IgG1a-Vybrant Blue (Miltenyi Biotec, Paris, France). On an average, $10^{4}$ cells were analyzed on either an Epics XL or Gallios cytometer (Beckman Coulter, Villepinte, France) and data were processed with the Expo 32 and Kaluza softwares, respectively (Beckman Coulter)

For cell cycle experiments, cells were washed with PBS and fixed in $70 \%$ ethanol $(\mathrm{EtOH}) / \mathrm{PBS}$ at $-20^{\circ} \mathrm{C}$ for at least $30 \mathrm{~min}$. Cells were then washed with PBS and resuspended in complete RPMl 1640 medium. Antibodies were added and the cells incubated for $30 \mathrm{~min}$ at $4^{\circ} \mathrm{C}$, washed with PBS and further incubated in PBS containing $10 \mu \mathrm{g} / \mathrm{ml}$ of propidium iodide and $100 \mu \mathrm{g} / \mathrm{ml}$ of RNAse A. On an average, $10^{4}$ cells were analyzed.

For $\gamma \mathrm{H} 2 \mathrm{AX}$ labeling, cells were stained as described in Huang et al. ${ }^{30}$ Briefly, washed cells were permeabilized in ice-cold $1 \%$ formaldehyde/PBS, incubated for $15 \mathrm{~min}$ on ice and fixed in $70 \% \mathrm{EtOH} / \mathrm{PBS}$ at $-20^{\circ} \mathrm{C}$. Cells were then blocked in $\mathrm{PBS} / 1 \%$ bovine serum albumin (BSA) $/ 0.2 \%$ Triton $\mathrm{X}-100$ for $5 \mathrm{~min}$ at room temperature, centrifuged and resuspended in PBS/1\% BSA $/ 0.2 \%$ Triton $\mathrm{X}-100$ containing anti- $\gamma$ H2AX-FITC antibody (Millipore, Billerica, MA, USA). Following an overnight incubation at $4{ }^{\circ} \mathrm{C}$, the cells were washed, resuspended in PBS containing RNAse A $(100 \mu \mathrm{g} / \mathrm{ml})$ and propidium iodide $(5 \mu \mathrm{g} / \mathrm{ml})$ and incubated for $30 \mathrm{~min}$ at room temperature, and then subjected to flow cytometry analysis. On an average, $5 \times 10^{3}$ cells were analyzed.

DCFDA (Invitrogen, Life Technologies, Saint-Aubin, France) staining was performed according to Cossarizza et al. ${ }^{31}$ On an average, $2 \times 10^{4}$ cells were analyzed.

Cell sorting. When cell viability was $<70 \%$, dead cells were removed using dead cell removal kit (Miltenyi Biotec) following the manufacturer's instructions, except that twice the recommended amount of beads was used.

CD138 ${ }^{\text {low }}$ cells were separated from CD138 $8^{\text {high }}$ cells using anti-CD138 antibody coupled to magnetic microbeads (Miltenyi Biotec) as described by Matsui et al. ${ }^{8}$

Migration assay. Cells were resuspended in RPMI 1640 containing $1 \%$ FCS at $10^{6}$ cells per $\mathrm{ml}$ and $100 \mu \mathrm{l}$ were placed in an insert with a pore size of $8 \mu \mathrm{m}$ (Millipore). Inserts were placed into 24-well plates filled with either the control medium (medium of untreated cells, i.e. cells treated with $0 \mathrm{nM}$ doxorubicin for 2 days) or the conditioned medium (medium of cells treated with $50 \mathrm{nM}$ doxorubicin for 2 days). Cells were incubated overnight and labeled with CD138 and propidium iodide to exclude any dead cells before flow cytometry analysis. On an average, $1.5 \times 10^{3}$ cells were analyzed.

Determination of cell senescence. Cells were either stained with $\beta$ galactosidase staining kit (Cell Signaling Technology, Beverly, MA, USA according to the manufacturer's instructions or with $\mathrm{C}_{12} \mathrm{FDG}$ as previously described in Debacq-Chainiaux et al. ${ }^{32}$ Briefly, cells were pre-treated for $1 \mathrm{~h}$ with $100 \mathrm{nM}$ bafalomycin $\mathrm{A} 1$ and further incubated for $2 \mathrm{~h}$ with $33 \mu \mathrm{M} \mathrm{C}{ }_{12} \mathrm{FDG}$ (a fluorogenic substrate of $\beta$-galactosidase). Cells were washed with PBS and analyzed by flow cytometry.

Clonogenicity assay. Cells were seeded in MethoCult (StemCell Technologie, Grenoble, France) according to the manufacturer's instructions. Cells $\left(10^{4}\right)$ in Iscove's MDM plus $2 \%$ FCS were seeded in methylcellulose in triplicate and incubated for 15 days. Colonies containing more than 40 cells were counted using an inverted microscope. For replating, cells were washed in PBS before being seeded.

Immunohistochemistry. Paraffin-embedded tumors were cut into $3-\mu \mathrm{m}$ thick slices. The paraffin was removed, antigens were unmasked in citrate buffe pH $6.4\left(10 \mathrm{~min}, 95^{\circ} \mathrm{C}\right)$ and immunolabeling was performed with anti-CD138 antibody (Dako France, Trappes, France). Labeling was revealed using the Vector Vectastain Elite kit (Vector Laboratories, Burlingame, CA, USA) and $3^{\prime}, 3^{\prime}$. diaminobenzidine (Dako France, Trappes, France). Slides were counterstained with hematoxylin.

Lentiviral infection. RPMI 8226 cells were infected with lentiviral particles containing shRNA directed against CHK2 (Open Biosystems, Huntsville, AL, USA) and selected with puromycin. Cells infected at the same $\mathrm{MOI}$ with lentiviral particles containing shRNA directed against glyceraldehyde 3-phosphate dehydrogenase (GAPDH) were used as control. At $72 \mathrm{~h}$ post-transduction, cells were analyzed by flow cytometry for GFP expression and 15 days later by western blot for CHK2 extinction.

Western blotting. Western blotting was performed as previously described in Roue et $a l^{33}$ The following antibodies were used: anti-GAPDH (Life Technologies SAS, Saint Aubin, France), anti-CHK2 and anti-pCHK2 (Thr68) (Cell Signaling Technology) and anti- $\gamma \mathrm{H} 2 \mathrm{AX}$ (Millipore). The cytokine assay was used according to the manufacturer's instruction (Panomics, Santa Clara, CA, USA).

QRT-PCR. Total RNA was extracted using Trizol reagent and cDNAs were generated using M-MLV reverse transcriptase according to the manufacturer's instructions (Life Technologies SAS). QRT-PCRs were performed using SYBR Green Master Mix with a SDS7000 apparatus (Applied Biosystems Inc., Courtaboeuf, France) according to the manufacturer's instruction. Standard curves were generated for each gene to determine PCR efficiencies, and relative gene expression was calculated.

Engraftment in nude mice. Irradiated or control cells $\left(10^{4}\right.$ cells) were mixed (v/v) with Matrigel (BD Biosciences, Le Pont de Claix, France) and injected subcutaneously into the flanks of 6-week-old Swiss nude female mice (Charles Rivers, Chatillon-sur-Chalaronne, France). Mice ( $n=5$ for each series) were monitored twice a week for tumor formation. At 12 weeks post-injection, tumors were visible and mice were killed. During experiments, mice were maintained in accordance with the principles of the Declaration of Helsinki and the French legislation on animal welfare. At the end of the experiment, tumors were excised and immediately fixed in 1\% paraformaldehyde, and then dehydrated and embedded in paraffin for immunohistochemistry.

Statistical analyses. Student's t-test was used to determine the significance of differences between experimental groups. Data were analyzed with two-tailed tests and $P<0.05$ was considered significant.

\section{Conflict of interest}

The authors declare no conflict of interest.

Acknowledgements. We thank A Barbaras, $\mathrm{P}$ Chevallier and $B$ Haelewyn for technical help; F Gouilleux, F Pflumio, A Ploubidou, R Harvey, M Ramialison and I Kronja for critical reading of the manuscript; the service of radiotherapy of the Center François Baclesse of Caen for the use of X-irradiation source, the SF 4206 (Université de Caen) for microscopy and flow cytometry facilities, the CURB (Université de Caen) for mice experiments and the Laboratory Frank Duncombe for letting us use their $\mathrm{P}^{+}{ }^{+}$laboratory. $\mathrm{JC}$ received post-doctoral fellowships from Conseil de Radioprotection d'EDF and Conseil Régional of Basse-Normandie; SB is supported by the Ministère de l'Enseignement Supétieur et de la Recherche. This work was supported by the Ligue contre le Cancer - Comité de l'Orne (to JC) and Comité du Calvados (to BS).

1. Clarke MF, Dick JE, Dirks PB, Eaves CJ, Jamieson CH, Jones DL et al. Cancer stem cells perspectives on current status and future directions: AACR Workshop on cancer stem cells. Cancer Res 2006; 66: 9339-9344.

2. Bleau AM, Hambardzumyan D, Ozawa T, Fomchenko El, Huse JT, Brennan CW et al. PTEN/PI3K/Akt pathway regulates the side population phenotype and ABCG2 activity in glioma tumor stem-like cells. Cell Stem Cell 2009; 4: 226-235.

3. Achuthan S, Santhoshkumar TR, Prabhakar J, Nair SA, Pillai MR. Drug-induced senescence generates chemoresistant stemlike cells with low reactive oxygen species. J Biol Chem 2011; 286: 37813-37829.

4. Bao S, Wu Q, McLendon RE, Hao Y, Shi Q, Hjelmeland AB et al. Glioma stem cells promote radioresistance by preferential activation of the DNA damage response. Nature 2006; 444: 756-760.

5. Diehn M, Cho RW, Lobo NA, Kalisky T, Dorie MJ, Kulp AN et al. Association of reactive oxygen species levels and radioresistance in cancer stem cells. Nature 2009; 458: 780-783.

6. Apple A. More shots on target. Nature 2011; 480: S40-S42.

7. Raab MS, Podar K, Breitkreutz I, Richardson PG, Anderson KC. Multiple myeloma. Lancet 2009; 374: 324-339. 
8. Matsui W, Huff CA, Wang Q, Malehorn MT, Barber J, Tanhehco Y et al. Characterization of clonogenic multiple myeloma cells. Blood 2004; 103: 2332-2336.

9. Pilarski LM, Seeberger K, Coupland RW, Eshpeter A, Keats JJ, Taylor BJ et al. Leukemic B cells clonally identical to myeloma plasma cells are myelomagenic in NOD/SCID mice. Exp Hematol 2002; 30: 221-228.

10. Van Valckenborgh E, Matsui W, Agarwal P, Lub S, Dehui X, De Bruyne E et al. Tumor-initiating capacity of CD138 - and CD138 + tumor cells in the 5T33 multiple myeloma model. Leukemia 2012; 26: 1436-1439.

11. Jakubikova J, Adamia S, Kost-Alimova M, Klippel S, Cervi D, Daley JF et al. Lenalidomide targets clonogenic side population in multiple myeloma: pathophysiologic and clinical implications. Blood 2011; 117: 4409-4419.

12. Rasmussen T, Haaber J, Dahl IM, Knudsen LM, Kerndrup GB, Lodahl M et al. Identification of translocation products but not K-RAS mutations in memory $B$ cells from patients with multiple myeloma. Haematologica 2010; 95: 1730-1737.

13. Matsui W, Wang Q, Barber JP, Brennan S, Smith BD, Borrello I et al. Clonogenic multiple myeloma progenitors, stem cell properties, and drug resistance. Cancer Res 2008; 68: 190-197.

14. Kastan MB, Bartek J. Cell-cycle checkpoints and cancer. Nature 2004; 432: 316-323.

15. D'Adda di Fagagna F. Living on a break: cellular senescence as a DNA-damage response. Nat Rev Cancer 2008; 8: 512-522.

16. Gire V, Roux P, Wynford-Thomas D, Brondello JM, Dublic V. DNA damage checkpoint kinase Chk2 triggers replecative senescence. EMBO J 2004; 23: 2554-2563.

17. Rodier F, Coppe JP, Patil CK, Hoeijmakers WA, Munoz DP, Raza SR et al. Persistent DNA damage signalling triggers senescence-associated inflammatory cytokine secretion. Nat Cell Biol 2009; 11: 973-979.

18. Coppe JP, Patil CK, Rodier F, Sun Y, Munoz DP, Goldstein j et al. Senescence-associated secretory phenotypes reveal cell-nonautonomous functions of oncogenic RAS and the p53 tumor suppressor. PLoS Biol 2008; 6: 2853-2868.

19. Krtolica A, Parrinello S, Lockett S, Desprez PY, Campisi J. Senescent fibroblasts promote epithelial cell growth and tumorigenesis: a link between cancer and aging. Proc Natl Acad Sci USA 2001; 98: 12072-12077.

20. Giuliani N, Bonomi S, Romagnani P, Lazzaretti M, Morandi F, Colla S et al. CXCR3 and its binding chemokines in myeloma cells: expression of isoforms and potential relationships with myeloma cell proliferation and survival. Haematologica 2006; 91: 1489-1497.

21. Karnoub AE, Dash AB, Vo AP, Sullivan A, Brooks MW, Bell GW et al. Mesenchymal stem cells within tumour stroma promote breast cancer metastasis. Nature 2007; 449: 557-563.

22. Kim D, Park CY, Medeiros BC, Weissman IL. CD19( - )CD45(low/ - )CD38(high)/CD138(+) plasma cells enrich for human tumorigenic myeloma cells. Leukemia 2012 (in press).
23. Liu D, Hornsby PJ. Senescent human fibroblasts increase the early growth of xenograft tumors via matrix metalloproteinase secretion. Cancer Res 2007; 67: 3117-3126.

24. Li F, Tiede B, Massague J, Kang Y. Beyond tumorigenesis: cancer stem cells in metastasis. Cell Res 2007; 17: 3-14.

25. Takacova S, Slany R, Bartkova J, Stranecky V, Dolezel P, Luzna P et al. DNA damage response and inflammatory signaling limit the MLL-ENL-induced leukemogenesis in vivo. Cancer Cell 2012; 21: 517-531.

26. Mani SA, Guo W, Liao MJ, Eaton EN, Ayyanan A, Zhou AY et al. The epithelialmesenchymal transition generates cells with properties of stem cells. Cell 2008; 133: 704-715.

27. Kawano M, Kuramoto A, Hirano T, Kishimoto T. Cytokines as autocrine growth factors in malignancies. Cancer Surv 1989; 8: 905-919.

28. Reynaud D, Pietras E, Barry-Holson K, Mir A, Binnewies M, Jeanne M et al. IL-6 controls leukemic multipotent progenitor cell fate and contributes to chronic myelogenous leukemia development. Cancer Cell 20: 661-673.

29. Axelrod R, Axelrod DE, Pienta KJ. Evolution of cooperation among tumor cells. Proc Natl Acad Sci USA 2006; 103: 13474-13479.

30. Huang X, Darzynkiewicz Z. Cytometric assessment of histone H2AX phosphorylation: a reporter of DNA damage. Methods Mol Biol 2006; 314: 73-80.

31. Cossarizza A, Ferraresi R, Troiano L, Roat E, Gibellini L, Bertoncelli L et al. Simultaneous analysis of reactive oxygen species and reduced glutathione content in living cells by polychromatic flow cytometry. Nat Protoc 2009; 4: 1790-1797.

32. Debacq-Chainiaux F, Erusalimsky JD, Campisi J, Toussaint O. Protocols to detect senescence-associated beta-galactosidase (SA-betagal) activity, a biomarker of senescent cells in culture and in vivo. Nat Protoc 2009; 4: 1798-1806.

33. Roué G, Pichereau V, Lincet H, Colomer D, Sola B. Cyclin D1 mediates resistance to apoptosis through upregulation of molecular chaperones and consequent redistribution of cell death regulators. Oncogene 2008; 27: 4909-4920.

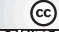

SOMERIEHISBESER

Cell Death and Disease is an open-access journal published by Nature Publishing Group. This work is licensed under the Creative Commons Attribution-NonCommercial-No Derivative Works 3.0 Unported License. To view a copy of this license, visit http://creativecommons.org/licenses/by-nc-nd/3.0/

Supplementary Information accompanies the paper on Cell Death and Disease website (http://www.nature.com/cddis) 